\section{Acoustics and metastable}

\section{liquids}

\section{Sébastien Balibar}

Laboratoire de Physique Statistique de l'Ecole Normale Supérieure associé au CNRS et aux Universités Paris 6 \& 7 24 rue Lhomond 75231 Paris Cedex 05, France

$M$ edical doctors know how to break kidney stones with ultrasound. We break pure liquids in a similar way. This has lead us to general questions on the metastability of liquid matter with respect to a gaseous or solid state.

\section{Acoustic cavitation}

Can one really break a liquid with ultrasound waves? Is this what happens in an ultrasonic cleaning bath? In such a device, the sound pressure oscillates strongly enough for bubbles to nucleate in the minima of the wave, and the subsequent collapse of these bubbles cleans walls. When evaporating into the gas bubbles, molecules separate from their neighbors in the liquid phase, so that, in a sense, one could say that the cohesion of the liquid is broken by the intense sound. Now, in this particular situation, the nucleation is called "heterogeneous" because it is not an intrinsic property of the liquid. In fact, micro-bubbles of air are already present in the bath, especially on wall defects. They grow under the effect of moderate depressions, and an ultrasonic cleaning bath does not require a very intense sound field. The liquid in this cleaning bath is fragile, like a solid piece which contains many micro-fractures.

Ocean water is just as fragile: it also breaks at moderately negative pressures, around - 1 bar. ${ }^{1}$ This is the reason why bubbles appear behind the propellers of fast moving boats. A local increase in flow velocity reduces the pressure: Bernoulli's law tells us that the quantity $\left(\mathrm{P}+1 / 2 \rho \mathrm{v}^{2}\right)$ is constant in an inviscid fluid ( $\mathrm{P}$ is the pressure, $\rho$ the density and $v$ the local fluid velocity). As a consequence, if v equals $15 \mathrm{~m} / \mathrm{s}$ somewhere, the pressure is reduced by 2 bar with respect to a fluid region at rest. This usually happens near the core of vortex lines behind each propeller blade, where the pressure is $1-2=-1$ bar. This is the typical negative pressure at which micro-bubbles of air grow in the ocean. Cavitation behind a submarine depends on the quantity and size of micro-bubbles which itself depend on depth, latitude, temperature etc. After growing in a

\footnotetext{
${ }^{1}$ Some people have difficulties in considering negative pressures, although there is not much mystery about them [1]. They are just positive stresses, and a pressure is nothing but an isotropic stress. If one pushes on a piston closing a chamber filled with some fluid, the pressure increases. On the contrary, if one pulls on the piston, one applies a positive stress that is a negative pressure. Of course, in a gas, the pressure cannot be negative; it tends to zero proportionally to the density (according to the perfect gas law). In a liquid, the pressure can be negative because of the existence of attracting forces between molecules. These forces ensure the cohesion of the liquid, i.e. a non-zero density at zero pressure. At negative pressure, matter is not stable but it can be metastable for very long times. This is the case of our liquid under extension: if bubbles appeared, they would grow and bring the liquid back to some positive pressure, but if no bubbles are present the stretched liquid state can stay for very long times. This may even happen in nature, for example at the top of a large tree, where the water in the trunk is stressed by the whole liquid column: the pressure is negative as soon as the height is larger than 10 meters.
}

region of negative pressure, bubbles move away and collapse in a region of positive pressure. This makes noise and may also damage the propeller blades, so that it is a serious technological problem.

Would it be possible to eliminate walls, defects, and impurities? What would happen then? This is in fact the motivation of our present research. We eliminate heterogeneous nucleation by focusing a sound wave in the bulk of an ultra pure liquid containing no dissolved gases. For this, we have started with the purest possible liquid, which is liquid helium because it is colder than any other one, so that all possible impurities are frozen and easy to filter. In this ideal situation, we observed "homogeneous" nucleation , an intrinsic property of the liquid. After about a decade of research, we have proven that the intrinsic tensile strength of liquid helium is $-9.4 \mathrm{bar}$ in the low temperature limit. This is close to the "spinodal limit" where the attractive forces responsible for the cohesion of the liquid can no longer stand the negative pressure of the acoustic wave.

Some people consider that liquid helium has been studied for too many years! In fact, we take advantage of this situation: the thermodynamic properties of liquid helium are so accurately known, in particular its equation of state $P(\rho)$ is so well established, that one could calculate the spinodal limit for the liquid-gas transition, and compare with the measured one. In our opinion, liquid helium is a remarkable model liquid.

For our measurements, we focus bursts of ultrasound waves, as was first done by J. Nissen et al. [2]. A hemispherical piezoelectric transducer is excited at resonance in its first thickness mode, near 1 $\mathrm{MHz}$. For our first experiments with M.S. Pettersen in 1994 [3], we used rather long bursts but we now use short ones [4,5], typically with 1 to 6 oscillations only. As a consequence of the geometry, the sound waves converge at the center, far from any wall (see Fig. 1). There, at the acoustic focus, we obtain typically 10 bar sound amplitude for bursts of 6 oscillations with 20 Volt excitation, enough to reach -9 bar if the static pressure in the cell is +1 bar. The accuracy of our measurements has continuously improved, and, together with F. Caupin [4], we have demonstrated the existence of a reproducible cavitation threshold at -9.4 bar in liquid helium at low

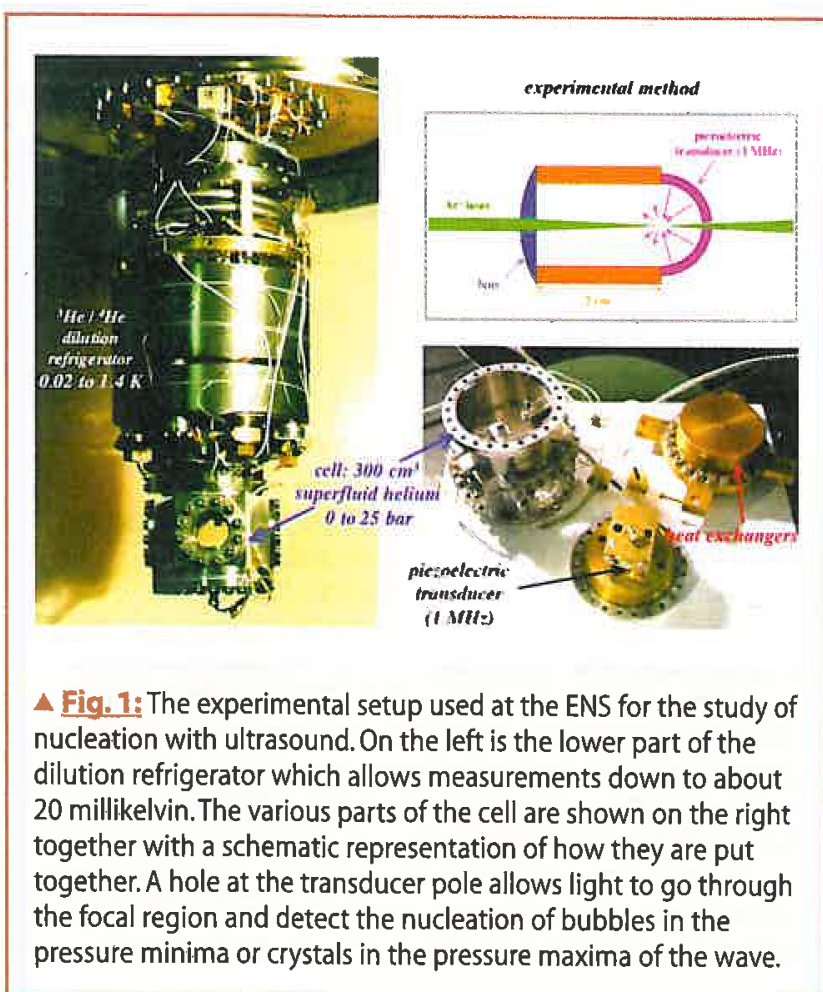


temperature. This is 0.2 bar only above the theoretical spinodal limit ( -9.6 bar) which was calculated by three different methods [6].

At this limit, the liquid is unstable because its compressibility diverges, it is infinitely soft and the sound speed vanishes. Not only is our measured value close to the theoretical value, in fact we understand this slight difference: -9.6 bar is the negative pressure at which liquid helium would be totally unstable, even if one observed it during an infinitely short time and in a vanishingly small volume. In our experiment, we look for cavitation in a time of order $100 \mathrm{~ns}$, i.e. a tenth of the sound period, and in a volume of about $10^{-7} \mathrm{~cm}^{3}$, a fraction of the acoustic wavelength cubed. This is why we find an instability at a slightly less negative pressure. In summary, we have shown with this model liquid that studying cavitation in clean conditions gives information on the internal cohesion of the liquid.

Is this another curiosity of helium, or could one use this method to learn something about other liquids? We are currently generalizing it to more classical liquids, in particular to the most important one, namely water, of course. Frederic Caupin and Vincent Fourmond [7] have first observed homogeneous cavitation in freon, then ethanol, and they are studying pure water now. The tensile strength of water is expected to be near -1500 bar at room temperature $\left(+35^{\circ} \mathrm{C}\right)$, and reaching the necessary sound amplitude is becoming a real challenge. Why is it so interesting to do this? It happens that, even at the beginning of the 21 st century

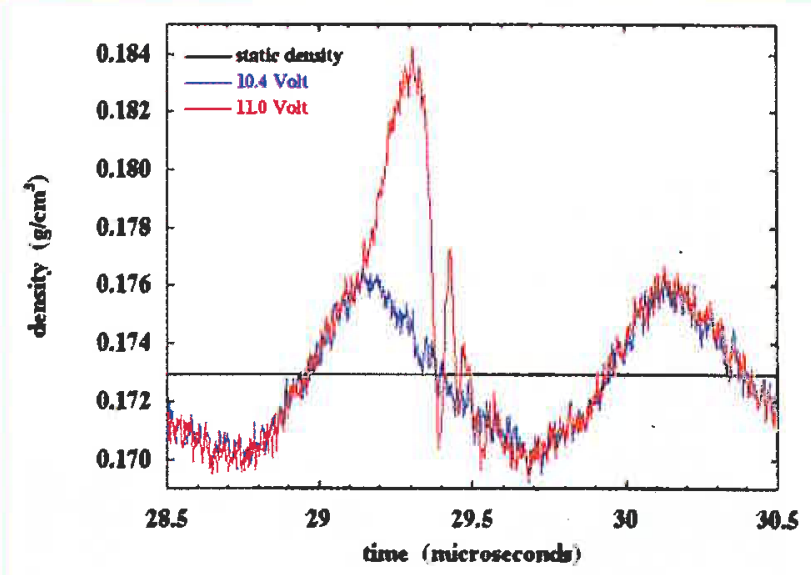

$\triangle$ Fig. 2: Acoustic crystallization on a glass plate. This recording from $X$. Chavanne et al. [5] shows a high amplitude wave propagating in liquid helium 4 (blue curve). With 11 Volt excitation, a pressure oscillation of \pm 4.3 bar is obtained at the acoustic focus, which corresponds to a $2 \%$ oscillation in density around the static density in the cell $\left(0,173 \mathrm{~g} / \mathrm{cm}^{3}\right)$. Beyond this threshold, liquid helium crystallizes, and one measures a sudden increase in density (red trace). Crystals can grow up to a size of 10 microns in $100 \mathrm{~ns}$, which means at velocities of $100 \mathrm{~m} / \mathrm{s}$, close to the speed of sound $(360 \mathrm{~m} / \mathrm{s})$. This recording was obtained when the acoustic wave was focused on a clean glass plate; it allowed a measurement of the instantaneous density from the reflectivity of light at the glass/helium interface in the acoustic focal region. where physics has reached a rather high level of power and complexity, the theory of water is not well established. F. Caupin expects his measurements to discriminate between two different theories, the one by R. Speedy et al. [8] and the one by S.Sastry et al. [9].

It is going to be a test because the temperature variation of the cavitation threshold is linked to the sign of the thermal expansion coefficient. One knows that, at ordinary pressure and below $4^{\circ} \mathrm{C}$, water expands when cooled down. This anomalous behavior comes from the fact that when $\mathrm{H}_{2} \mathrm{O}$ molecules cool down, they bind to each other thanks to "hydrogen bonds", and a local symmetry is forced, which is tetrahedral, i.e. not compact; as a consequence, $\mathrm{H}$-bonds take space. Now does this expansion occur in stretched water as well, i.e. at large negative pressure, or not? This is where Speedy and Sastry disagree. If Speedy is right, then the tensile strength of liquid water should decrease with temperature; but if Sastry is right, it should increase. It thus seems that Caupin's measurement should easily tell the truth; we hope to know soon.

One reason why Caupin is optimistic is that he also studied the light isotope of helium, helium 3 , and he has already observed a similar effect. He first observed that liquid helium 3 is three times more fragile than liquid helium 4 (it "breaks" at -3.1 bar instead of -9.4). He then observed an anomalous temperature variation of its cavitation threshold pressure: below $0.3 \mathrm{~K}$, it decreases with temperature, and he could relate this to the thermal expansion coefficient being negative; it is negative because helium 3 is a Fermi liquid where the effective mass of quasiparticles increases with pressure, a reason which has nothing to do with $\mathrm{H}$-bonds in water, but the consequence on cavitation is the same, and Caupin could observe it. He only has now to focus acoustic waves of 1500 bar amplitude. This corresponds to $1.5 \mathrm{MW} / \mathrm{cm}^{2}$ or $220 \mathrm{~dB}$, a very large power density, about 20000 times more than that used by surgeons to break kidney stones. If the physics of helium could lead us to learn something crucial about water, this would of course be a great success. But this is far from being the only question of interest to us, when playing with high intensity acoustic waves.

\section{Acoustic crystallization}

What about the pressurization during the positive swings which immediately follow the negative ones in the acoustic waves? Could it be sufficiently strong to crystallize the liquid in the path of the wave? The answer is yes, and we observed this phenomenon for the first time in 2001 [5]. The physics is similar but the nucleation of crystals is usually favored by walls. In all practical situations, crystals nucleate on walls or have not enough time to grow during one sound period, and that's probably why acoustic crystallization had not yet been observed. To eliminate the influence of walls is clearly difficult, although it has been done by G. Seidel and his colleagues, who studied the crystallization of droplets of liquid hydrogen [10].

But let us first consider the case of water again. One knows that liquid water can be supercooled below $0^{\circ} \mathrm{C}$. For example, in clouds, the liquid droplets freeze around $-20^{\circ} \mathrm{C}$, a temperature that depends on pollution. Now, it has been a long standing puzzle to understand why water could never be supercooled below $-40^{\circ} \mathrm{C}$. It seems that Peter Taborek has solved this problem with his 1985 experiment [11]. According to the standard theory of nucleation, it is the value of the surface tension that governs the limit beyond which a liquid can no longer stay in its metastable supercooled state. For a nucleus of the stable state to appear, a liquid-solid interface needs to be formed, whose energy is proportional to the surface tension, a free energy per unit area. According to Taborek, the free energy of the ice-to-water interface is $28 \mathrm{erg} / \mathrm{cm}^{2}$, and this explains the maximum undercooling of liquid water. Now, measuring the surface tension of a crystal is far from easy, and the value 


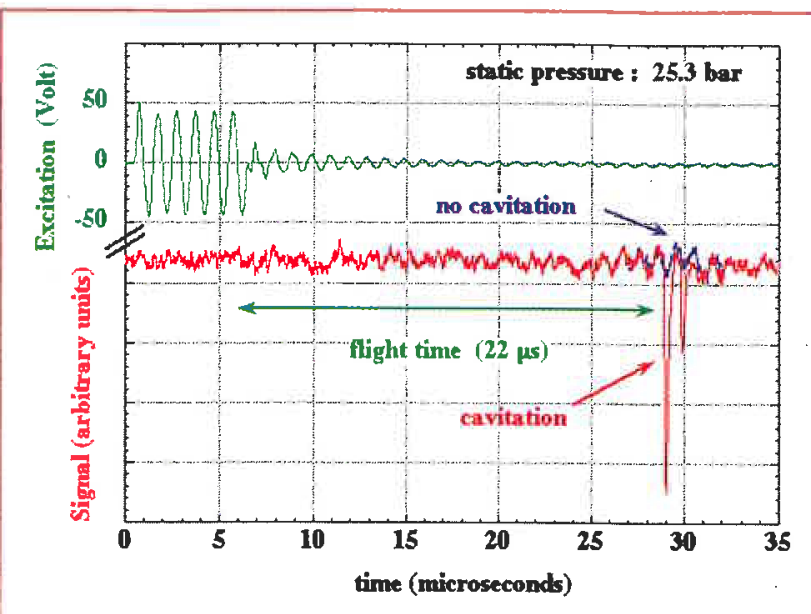

A Fig. 3: Homogeneous acoustic cavitation. In liquid helium at high pressure and far away from any wall, a high intensity acoustic wave-here 35 bar in amplitude-produces cavitation in its negative swings, no crystallization in its positive swings. This cavitation is random, and recordings at the threshold show cavitation with a probability 0.5 . One easily distinguishes a cavitation signal from a crystallization signal by studying the dependence of the phenomenon on the static pressure in the experimental cell.

of this quantity was not known for ice. Taborek's value is reasonable and probably right, but so far there seems to have been no independent check that his value is correct, so that the whole issue is still somewhat controversial.

What about helium, then? Would it be possible to use it once more as a model system to understand the metastability limit of a liquid against its crystallization? This is exactly what we are presently doing, and we take advantage of the fact that, in the case of helium crystals, the surface tension has been accurately measured thanks to the possibility of propagating crystallization waves at their surface [12].

A first important step was the recent observation of crystallization by acoustic waves on a clean glass plate [5]. This plate allowed a local and instantaneous measurement of the density at the acoustic focus, from the reflectivity of light at the glass/helium interface (Figure 2). We found that helium crystals nucleated 4.3 bar above the equilibrium pressure between solid and liquid helium (25.3 bar). This overpressure was about 1000 times larger than what had been observed during ordinary experiments in ordinary cells, namely a few millibar. This we understood in that, in ordinary cells, favorable nucleation sites existed, such as graphite dust particles probably [13], while our clean glass plate had none of these dust particles. Still, from the value of the surface tension of helium crystals, it has been predicted that homogeneous nucleation of helium crystals should occur around 65 bar. We thus guessed that the glass plate favored the nucleation, probably because of the van der Waals attraction it exerts on liquid helium, and of the presence of a few irregularities at its surface that may amplify the effect of the van der Waals attraction. We verified this hypothesis by measuring the energy barrier for the nucleation of crystals on the glass plate and also the number of nucleation sites, which we found of order one. Given this, we removed the glass plate in our setup (Fig. 1) and continued our search for homogeneous nucleation of helium crystals.

As in the previous experiment, a green laser was focused in the acoustic focal region. Since we had shown that the light scattering technique was sensitive enough to detect the nucleation of crystals in the presence of a glass wall, there was no reason to think that, in the absence of glass wall, we could miss nucleation events. Moreover, when using higher amplitude waves, the crystals should grow even faster and be larger and easier to see. As already mentioned, the standard nucleation theory predicts homogeneous crystallization at 65 bar. We reached this overpressure, then reached 110 bar, finally 160 bar at the end of 2003 [15], but we never detected any crystallization, only cavitation in the negative swings of the wave (see Fig. 3).

A first consequence of our experimental observations is of course that the standard theory fails in predicting the nucleation threshold for helium crystals from overpressurized liquid helium. It is clearly too simple to predict the properties of liquids that far from their stable region of existence. For example, in order to describe the energy of the nucleus surface, it uses the value of the liquid-solid interfacial tension at the equilibrium pressure, where the respective densities of the liquid and the solid are 0.17 and $0.19 \mathrm{~g} / \mathrm{cm}^{3}$, while the density of liquid helium at 160 bar is larger than $0.22 \mathrm{~g} / \mathrm{cm}^{3}$. F. Caupin and H.J. Maris have proposed that the liquid-solid interfacial tension increases with pressure, so that the solid should never nucleate, except if one reaches some instability at a certain critical pressure. Could this happen?

A long time ago, Schneider and Enz [16] proposed that liquid helium should be unstable against the formation of a periodic state if the "roton gap" vanished. The rotons are the particular excitations that were introduced by Landau to explain the thermodynamics of superfluid helium. They correspond to a local minimum in the dispersion relation $\omega(\mathrm{q})$ of phonons, and, since Landau's time, one has understood that the roton energy reflects the local order of the liquid around the atoms. As the pressure increases, the local order increases and the roton energy decreases, and this has been very accurately measured by successive neutron scattering measurements. The roton wavevector q being roughly equal to $2 \pi / a$ where a is the interatomic distance, the liquid is expected to be unstable against the formation of a periodic state whose periodicity is the atomic distance, and this looks very close to a crystal. As a consequence, one expects a liquid-solid spinodal to exist at the pressure where the roton energy vanishes, and this has been recently predicted to occur around $200 \mathrm{bar}$ [17]. We are not far from this possible limit, and we hope to reach it soon, for example by using two hemispherical transducers facing each other, instead of one. An observation of this instability would be a threedimensional analog of the one occuring in two dimensions, at the surface of ferrofluids where a surface mode with finite wavevector also becomes soft, this time as a function of the applied magnetic field.

Now, the possibility that the free energy of the liquid-solid interface increases with pressure is not the only possible explanation of our recent experiments. We have shown that, if the liquid is superfluid, then the crystals grow very fast after their nucleation, so that their observation is easy. But is liquid helium superfluid when its density is $30 \%$ larger than at equilibrium? If a superfluid to normal transition occurs, liquid helium might be very viscous at 160 bar and its dynamics jammed as in a glass. In fact this is certainly what happens with ordinary liquids after a high pressure quench. The question is thus: is a system of Bose spheres necessarily superfluid at zero temperature? If not, it might explain why we did not observe the crystallites: they don't grow and they are too small to be detected. This last question is rather open but has already triggered several theoretical works, by P. Nozières and by D. Ceperley et al. If we could observe nucleation near the instability at $200 \mathrm{bar}$, we should see some efffect of a superfluid to normal transition on 
the temperature variation of the nucleation limit at high pressure, as we did at low pressure for the cavitation limit.

It seems that, by repeatedly asking naive questions on the effect of high intensity acoustics in both liquid helium and water, on surface tensions and on the way that stable nuclei appear when matter changes state, we have encountered several unsolved problems. We have found a few answers to several questions, but a lot remains to be understood.

\section{About the author}

Sebastien Balibar leads a research group at the ENS in Paris. His main achievements concern roughening and other surface porperties of crystals, quantum evaporation, wetting and critical phenomena, nucleation of bubbles and crystals using acoustic waves.

e-mail: balibar@lps.ens.fr

http://www.lps.ens.fr/ balibar/

\section{References}

[1] H.J. Maris and S. Balibar, Negative pressures and cavitation in liquid helium, Physics Today 53, 29 (2000)

[2] J. Nissen, E. Bogedom, L.C. Brodie, and J.S. Semura, Phys. Rev. B 40, 6617 (1989).

[3] M.S.Pettersen, S.Balibar and H.J.Maris , Phys. Rev. B49, 12062 (1994).

[4] F. Caupin et S. Balibar, Phys. Rev. B64, 064507 (2001)

[5] X. Chavanne, S.Balibar and F. Caupin, Phys. Rev. Lett. 86, 5506 (2001); X. Chavanne, S.Balibar and F. Caupin, J. Low Temp. Phys. 125,155 (2001).

[6] For a general review, see S. Balibar, "Nucleation in quantum liquids", J. Low Temp. Phys. 129, 363 (2002)

[7] F. Caupin and V. Fourmond, in " Liquids under negative pressure, NATO conference ed, by A.R. Imre et al., p.307 (Kluwer Ac. Publishers, 2002).

[8] R. Speedy, J. Phys. Chem. 86, 982 (1982) et 86, 3002 (1982).

[9] S. Sastry, P.G. Debenedetti, F. Sciortino, and H. Stanley, Phys. Rev. E53, 6144 (1996).

[10] G. Seidel, H.J. Maris, FI.B. Williams and J.G. Gardon, Phys. Rev. Lett. 56,2380 (1986).

[11] P. Taborek, Phys. Rev. B32, 5902 (1985).

[12] K.O. Keshishev, A. Ya. Parshin and A. V. Babkin, Pis'ma Zh. Eksp. Teor. Fiz., 30, 63 (1979) [JETP Lett. 30, 56 (1980)] ; E.Rolley, E.Chevalier, C.Guthmann and S.Balibar, Phys. Rev.Lett. 72, 872 (1994) ; for a review, see S. Balibar, H. Alles and A. Ya. Parshin, Rev. Mod. Phys. (to appear in 2004).

[13] S.Balibar, T.Mizusaki and Y.Sasaki, J. Low Temp. Phys. 120, 293 (2000).

[14] F. Caupin, S. Balibar and H.J. Maris, Physica B, 329-333, 356 (2003)

[15] F.Werner, G. Beaume, A. Hobeika, S. Nascimbene, C. Herrmann, F. Caupin and S. Balibar, to appear in J. Low Temp. Phys. (2004).

[16] T. Schneider and C.P. Enz, Phys. Rev. Lett. 27, 1186 (1971).

[17] H.J. Maris obtained this rough estimate by using a density functional theory of liquid helium introduced by F. Dalfovo, A. Lastri, L. Pricaupenko, S. Stringari, and J. Treiner, Phys. Rev. B 52, 1193 (1995).

\section{Shining light on electron quantum liquids in two dimensions}

\author{
Vittorio Pellegrini ${ }^{1}$ and Aron Pinczuk $k^{2,3}$ \\ ${ }^{1}$ NEST-INFM and Scuola Normale Superiore, Pisa, Italy; \\ ${ }^{2}$ Dept. of Applied Physics and Applied Mathematics and Dept. of \\ Physics, Columbia University, New York, USA; \\ ${ }^{3}$ Bell laboratories, Lucent Technologies, Murray Hill, New Jersey, USA
}

7 the itinerant carriers that play pivotal roles in many of the propconstituent atoms. A simple assembly of itinerant electrons at densities of conventional metals behaves as a liquid of fermions that supports well-known electrical transport properties and collective behaviour with excitations such as plasma waves. The early work of E.P. Wigner and F. Bloch created an awareness of the intricate many-electron physics that may emerge when reaching the lowdensity limit [1]. As the electron density reduces, new highly correlated phases are expected as quantum mechanical interactions dictate key behaviours of the electron assembly. The difficulties in achieving the required low critical values of the density in high quality systems with very long electron relaxation times hinder the manifestation of broken-symmetry phases such as the electron crystal and ferromagnetic state proposed by Wigner and Bloch.

The endeavours linked to the creation, study and manipulation of new collective electron phases are among the most central areas of contemporary condensed-matter science. A key development in these branches of fundamental and applied science, that continues to have a major impact on current research, was the introduction of modulation-doped semiconductor quantum structures at the end of the 70's [2]. This advance in fabrication, by Molecular Beam Epitaxy (MBE) methods [see Figure 1a], enables creation of electron fluids in two dimensions (2D) in which scattering due to imperfections is highly suppressed to levels below those of intrinsic mechanisms (phonons) even at the lowest temperatures. The availability of nearly perfect electron systems in modulation-doped artificial quantum structures has stimulated the phenomenal expansion of studies of low-dimensional systems that continues to the present [3].

The discoveries of the integer and fractional quantum Hall effect are key milestones in condensed matter sciences [4,5]. The search for the elusive electron-crystal state in modulation-doped quantum structures led to the unexpected discovery of fractional quantization of the Hall effect of the two-dimensional electron gas in a perpendicular magnetic field [5]. The transport signatures of the fractional quantum Hall effects are seen as macroscopic manifestations of a quantum fluid in which the highly correlated electron liquid is represented by a quantum state with a single many-particle wave function [6]. Experimental and theoretical studies of quantum Hall electron liquids have uncovered some of the more intriguing many-body behavior in contemporary physics. The multiplicity of quantum Hall states seen in superior quality systems at low temperatures [see Figure 1d] can be regarded as sequences of 'compressible' (metallic) and 'incompressible' (insulating) states that occur as the external 